\title{
Functional Principles of Registry-based Service Discovery
}

\author{
V.Sundramoorthy ${ }^{1}$, C.Tan ${ }^{2}$, P.H.Hartel ${ }^{1}$, J.I.den Hartog ${ }^{1}$, and J.Scholten ${ }^{1}$ \\ ${ }^{1}$ University of Twente, Enschede, The Netherlands \\ \{vasughi, pieter, hartogji, scholten\}ecs.utwente.nl \\ ${ }^{2}$ Massachusetts Institute of Technology (MIT), Cambridge, USA \\ c.tan@mit.edu
}

\begin{abstract}
As Service Discovery Protocols (SDP) are becoming increasingly important for ubiquitous computing, they must behave according to predefined principles. We present the functional Principles of Service Discovery for robust, registry-based service discovery. A methodology to guarantee adherence to these principles is provided and illustrated by formal verification of the principles against $F R O D O$, an SDP built for the home environment. We show that, to make behavioral guarantees, an SDP has to be robust against network disturbances, and cannot rely only on the network layer.
\end{abstract}

Keywords: Service discovery protocols, Service Discovery Principles, Formal verification

\section{INTRODUCTION}

In the pursuit of a ubiquitous world, network administration, as we know it will change. Self-configuring systems will replace user configuration, where devices discover their environment, detect and adapt to topology changes, establish communication with each other and share services. Several state of the art systems have been developed towards achieving this objective, including Jini [1] by Sun Microsystems, Universal Plug and Play (UPnP) [2] by Microsoft, Service Location Protocol [3], [4] by the IETF and Bluetooth SDP [5] by the Bluetooth SIG consortium.

In general, service discovery protocols adhere to certain fundamental attributes. To start with, there are two types of entities in the system: User and Manager. A Manager is a service provider, which has a set of services. Each service is represented as a Service Description (SD), which describes the service in terms of: (1) device type (e.g. printer), (2) service type (e.g. color printing) and (3) attribute list (e.g. location, paper size). A User is an entity that has a set of requirements for the services it needs.

There are two types of service discovery architectures: registry-based (e.g. Jini) and peer-to-peer (e.g. UPnP). A registry-based architecture has a third entity, called the Registry. A Manager registers its services at a Registry, and Users discover the services through unicast queries to the Registry. In the peer-to-peer architecture there are no Registries, and Users discover Managers through broadcast or multicast queries. The registry-based architecture reduces network traffic and makes a network more manageable by allowing Registries to keep track of arriving and departing services. The peer-to-peer architecture avoids single point of failure problems, as may exist in the registry-based architecture, but increases network traffic. A combination of these two architectures can be implemented to allow the protocol to be more resilient against failure on the registry, and reduce network traffic (e.g. SLP, FRODO). However, unlike existing protocols, FRODO [6] implements resource-awareness. The service discovery tasks are partitioned according to the node's resource constraints, where a resourcelean node depends on more powerful neighbors to complement its discovery tasks. From this point onwards, we focus on protocols which include a Registry because the peer-to-peer architecture is a simplification of the registry-based architecture, and can be derived by removing Registry-related factors from this work.

Service discovery protocols perform garbage collection of defunct services. A popular mechanism for garbage collection is leasing. The Manager refreshes a "lease" periodically to notify the Registries of its continuous existence. The services of Managers that have left the network can be purged automatically if the lease is not renewed.

Most service discovery protocols also implement update functionalities for Managers to inform interested Users, whenever their services change. The change can either be reflected directly in the SD, or in an evented variable that gives the status of the service. Update mechanisms ensure subscribed Users remain consistent with the Managers whenever a service changes.

Contribution: The contributions of this paper to the field of service discovery are (1) stating for the first time, the functional principles for achieving robust registry-based service discovery, (2) classification of the functions in service discovery, (3) presenting a methodology for verifying service discovery protocols 
and (4) illustrating the above using our resource-aware and robust FRODO protocol.

Section 2 classifies service discovery mechanisms into four major functions, and the principles that correspond to each of the functions. Section 3 provides a brief description of FRODO. Section 4 describes the modeling and verification methodology of FRODO using DT-Spin, and Section 5 analyzes the results of verification. The last section concludes.

\section{The Principles of Service Discovery}

Service discovery systems that aim to be selfconfiguring should provide reliability in the face of network disturbances. This basically means that the service discovery functions are able to recover from errors. A system is flawed if a User is never able to discover a Manager in the system whose services match the User's requirements Thus, we feel it is imperative for the field of service discovery to have a set of principles that makes the notion of correct behavior precise. We call these Service Discovery Principles. Besides ensuring reliability, these fundamental principles define the nature and constraints of service discovery. Systems that adhere to the principles will provide guarantees on their capabilities and behaviors.

\section{A. Related Work}

The only other work done in this area is the Service Guarantees from NIST in [7], which proposes general guarantees that service discovery protocols should strive to satisfy. Our Service Discovery Principles refine these guarantees and moreover, we provide a methodology to proof using model-checking techniques, that a protocol satisfies these principles.

\section{B. Service Discovery Functional Areas}

Logically, service discovery is divided into 4 functional areas:

1) Configuration Discovery - Allows registry-based systems to (a) auto-configure a Registry and (b) discover the Registry. Auto-configuration of Registries require nodes to appoint Registries, according to some criteria, on the fly, without user interventions. For Registry discovery, nodes can actively initiate the discovery or listen lazily to Registry announcements.

2) Service Registration - Allows Managers to register their services at a Registry. Registration mechanisms include (a) unsolicited registration, where nodes request the Registry to register their services and (b) solicited registration, where Registries detect and request new nodes to register.

3) Service Discovery - Allows Users to discover Managers that satisfy their set of requirements. This could be achieved through a (a) unicast query to a Registry, (b) broadcast query, (c) notification of new services by the Registry to the Users or broadcast announcements of Managers of their services to the Users.

4) Configuration Update - After a User discovers a service, it can subscribe to the Manager to receive updates when the service changes, or becomes unavailable. The Managers provide two types of update information, service description update and evented variable update. The updates can be propagated using (a) 2-party scheme, where the communication is directly between the Manager and the User, or (b) 3-party scheme, where a registry propagates the update to the User on behalf of the Manager. Garbage collection of defunct services is also part of the update functionality.

Different service discovery systems implement different mechanisms to achieve the objectives of the functional areas, as depicted in Table I.

\section{The Service Discovery Environment}

During the operations of service discovery tasks, the system could experience various network disturbances, such as link failure, interface failure, message loss or node failure. Network disturbances cause uncertainties in service discovery systems. However, it is important to ensure that a service discovery system guarantees recovery from a disturbance.

Below we provide a formal description of a system.

a) System: A system consists of a set of entities, $(e \in) E$ with attributes, a set of services $(s \in) S$ and time in progress $(t \in)$ Time. The attributes of the entities, described below, evolve over time. Based on the attributes, the entities are divided into three (not necessarily pairswise disjoint) sets $(u \in) U$ for Users, $(\mathrm{m} \in) \mathrm{M}$ for Managers, and $(\mathrm{c} \in) \mathrm{C}$ for Registries.

b) Entity attributes: Each entity, e has the following attributes, which are subject to change over time:

i) $\mathrm{C}(\mathrm{e}) \subseteq \mathrm{E}$ is the set of Registries discovered by e. An entity is called a Registry, i.e. e $\in C$, if it has discovered itself in the role of Registry, $e \in C(e)$.

ii) OfferedSD(e) $\subseteq \mathrm{S}$ is the set of services, offered by e. An entity is called a Manager, i.e. e $\in M$, if it offers at least one service, OfferedSD $(e) \neq \emptyset$.

iii) Requirement $(e) \subseteq S$ is the set of services required by entity e. An entity is called a User, i.e. e $\in$ $\mathrm{U}$, if it is interested in/requires at least one service, Requirement $(\mathrm{e}) \neq \emptyset$.

iv) DiscoveredSD $\left(e, e^{\prime}\right) \subseteq S$ is the set of services discovered by e at entity $e^{\prime}$. This attribute is (typically) only used for e, a User and $\mathrm{e}^{\prime}$, a Manager. We put DiscoveredSD $(\mathrm{u}):=\bigcup$ DiscoveredSD $(\mathrm{u}, \mathrm{m})$

for the set of all services discovered by a User, $u$ at any Manager, $\mathrm{m}$.

v) RegisteredSD(e) $\subseteq \mathrm{S}$ is the set of registered services in e. This attribute is only used for a Registry. 


\begin{tabular}{|c|c|c|c|c|}
\hline $\begin{array}{l}\text { Functional Area, re- } \\
\text { lated Principles and } \\
\text { supportive attributes }\end{array}$ & Mechanisms & SLP & Jini & FRODO \\
\hline \multirow{2}{*}{$\begin{array}{l}\text { Configuration } \\
\text { Discovery (Registry } \\
\text { Setup and Registry } \\
\text { Discovery Principles) }\end{array}$} & $\begin{array}{l}\text { Auto-configured Reg- } \\
\text { istry }\end{array}$ & No & No & $\begin{array}{l}\text { Yes - through leader elec- } \\
\text { tion among } 300 \mathrm{D} \text { nodes }\end{array}$ \\
\hline & Registry Discovery & $\begin{array}{l}\text { Active and lazy discov- } \\
\text { ery }\end{array}$ & $\begin{array}{l}\text { Active and lazy discov- } \\
\text { ery }\end{array}$ & Active and lazy discovery \\
\hline $\begin{array}{l}\text { Registration (Regis- } \\
\text { tration Principle) }\end{array}$ & $\begin{array}{l}\text { Registration } \\
\text { mechanism }\end{array}$ & Unsolicited registration & Unsolicited registration & $\begin{array}{l}\text { Solicited and unsolicited } \\
\text { registration }\end{array}$ \\
\hline \multirow{2}{*}{$\begin{array}{ll}\text { Service } & \text { Discovery } \\
(\text { Service } & \text { Discovery } \\
\text { Principle }) & \end{array}$} & Search mechanism & $\begin{array}{l}\text { Unicast (multicast possi- } \\
\text { ble) }\end{array}$ & Unicast & $\begin{array}{l}\text { Unicast (multicast possi- } \\
\text { ble) }\end{array}$ \\
\hline & $\begin{array}{l}\text { Notification of new } \\
\text { services to Users }\end{array}$ & No & $\begin{array}{l}\text { Yes - unicast notification } \\
\text { by Registry }\end{array}$ & $\begin{array}{l}\text { Yes - unicast notification } \\
\text { by Registry }\end{array}$ \\
\hline \multirow[t]{3}{*}{$\begin{array}{l}\text { Configuration } \\
\text { update (2-party and } \\
\text { 3-party Configuration } \\
\text { Update Principles) }\end{array}$} & Garbage collection & Managers renew lease & Managers renew lease & $\begin{array}{lrr}300 \mathrm{D} & \text { Managers } & \text { renew } \\
\text { lease, } & \text { Registry } & \text { polls } \\
\text { 3D/3C } & \text { Managers } & \end{array}$ \\
\hline & Update information & Service update & Service update & $\begin{array}{l}\text { Service update, event noti- } \\
\text { fication }\end{array}$ \\
\hline & Update mechanism & None - register again & 3-party subscription & $\begin{array}{l}\text { 2-party subscription, 3- } \\
\text { party subscription }\end{array}$ \\
\hline \multirow[t]{4}{*}{$\begin{array}{l}\text { Supportive } \\
\text { attributes }\end{array}$} & Failure response & TCP-dependent & TCP dependent & $\begin{array}{l}\text { Retransmission, } \\
\text { acknowledgement, Backup } \\
\text { for Central, Polling, } \\
\text { Leader election }\end{array}$ \\
\hline & Resource awareness & No & No & Yes, device classification \\
\hline & Service usage & Application layer & Mobile proxy code & Application layer \\
\hline & Security & $\begin{array}{l}\text { Limited, with applica- } \\
\text { tion driven authetication }\end{array}$ & $\begin{array}{l}\text { None, depends on JDK } \\
1.2 \text { security model }\end{array}$ & None, future work \\
\hline
\end{tabular}

TABLE I

Taxonomy of registry-based service discovery systems. FRODO classifies devices into 3C, 3D and 300D, in the order of increasing resources. Service discovery tasks are partitioned according to resource constraints

There are several protocol-dependent parameters which are used in the Service Discovery Principles:

a) Connectivity condition: Conn $\left(e, e^{\prime}\right)$ : The service discovery protocol is responsible for providing the definition of Connectivity. An example is "if a message is transmitted from either e to e', or vice versa, the message is received.". The Connectivity condition is not restricted to valid communication paths between 2 entities. It can also be defined by an application, for example, a security application that detects a malicious entity, and indicates to the service discovery protocol that the node is not available for any further operations.

b) Disconnect condition:

$\operatorname{DisConn}\left(e, e^{\prime}\right)$ : $\neg$ Conn $\left(e, e^{\prime}\right)$ for "sufficiently long", where "sufficiently long" is a protocoldependent parameter. The definition of "sufficiently long" includes the Connectivity definition and the period communication did not occur. Examples are the limit of retransmissions or the time period for waiting for acknowledgements.

c) Global Connectivity, GC: This condition is satisfied if all entities are connected.

$\mathrm{GC}: \forall \mathrm{e}_{1}, \mathrm{e}_{2} \in \mathrm{E}: \operatorname{Conn}\left(\mathrm{e}_{1}, \mathrm{e}_{2}\right)$

d) N, the desired number of Registries in the system.

e) $\mathrm{G}(\mathrm{e})(\subseteq \mathrm{E})$ the Registries required by e. An entity may not be interested in knowing all Registries but only those of a certain type, i.e. those which satisfy its "Registry requirements".

f) Rank, a function for electing the Registries in the system, where the set $C$ has highestRank if $\neg\left(\exists \mathrm{e}^{\prime} \notin \mathrm{C}: \operatorname{Rank}\left(\mathrm{e}^{\prime}\right)>\min \{\operatorname{Rank}(\mathrm{e}) \mid \mathrm{e} \in \mathrm{C}\}\right)$

\section{Service Discovery Principles}

We use Linear Temporal Logic (LTL) [8] to define the Service Discovery Principles. This is essentially proportional logic with temporal operators such as $\square$ (always) or $\diamond$ (eventually). Basically the recovery of a service discovery system is defined as the response pattern, $\square\left(p^{\prime} \rightarrow \diamond p\right)$, where $p^{\prime}$ is the state of Connectivity, Global Connectivity or Disconnect, and $p$ is the response of the service discovery system towards satisfying a Service Discovery Principle.

We need some auxiliary definitions before giving the Service Discovery Principles.

a) The symbols " $\vee \subseteq$ ", " $\diamond \supseteq$ " and " $\vee \supset \subset$ "

$\mathrm{a} \diamond \subseteq \mathrm{b}$ holds at time $\mathrm{t}_{0}$ when a at time $\mathrm{t}_{0}$ is a subset of $b$ at some time $t_{1}$ where $t_{1} \geq t_{0}$. Similiarly, for the symbols " $\diamond \supseteq$ " and " $\diamond \supset \mathcal{C}$ " where $a \supset$ b denotes that the two sets are disjoint $(a \cap b=\emptyset)$.

b) ServiceSearch $(u, c)$ states that a User, $u$ is looking for a specific service from a Registry, $c$

c) Matching $\mathrm{SD}(\mathrm{c}, \mathrm{u}):=$

RegisteredSD $(c) \cap$ Requirement $(u)$ 


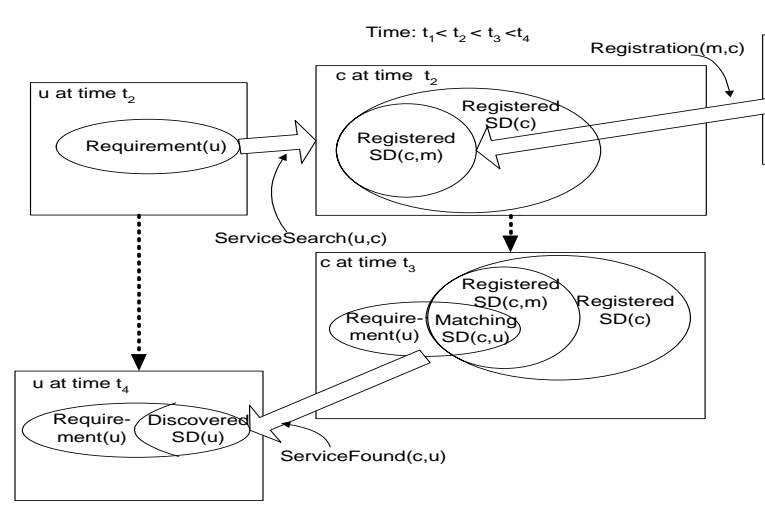

Fig. 1. System flow and relations between sets during Registration and Service Discovery. Registration, ServiceSearch and ServiceFound are shown as messages sent between entities. A set of service is registered by the Manager at time $t_{1}$, then discovered by the Registry at $t_{2}$, and User searches for the service at or before $t_{2}$. The Registry has processed the request at $\mathrm{t}_{3}$, where it finds some of the services provided by the Manager matching the requirements of the User. The User discovers the service at $t_{4}$.

is the set of services registered at the Registry, c which match the requirements of User, $u$.

d) ServiceFound $(c, u):=$

MatchingSD $(\mathrm{c}, \mathrm{u}) \diamond \subseteq$ DiscoveredSD $(\mathrm{u})$ states that the matching services at a Registry $c$ are discovered by the User $u$.

e) Registration $(m, c):=$

OfferedSD $(\mathrm{m}) \diamond \subseteq$ RegisteredSD (c)

states that all services of Manager, $m$ are registered at Registry, c.

f) $\operatorname{PurgeService}(\mathrm{m}, \mathrm{u}):=$

OfferedSD $(\mathrm{m}) \diamond \supset$ DiscoveredSD $(\mathrm{u})$

states that the services of Manager, $m$ are eventually purged from the discovered services of User, $u$.

PurgeService $(\mathrm{m}, \mathrm{c}):=$

OfferedSD $(\mathrm{m}) \diamond \supset$ RegisteredSD $(\mathrm{c})$

similarly states that the services of Manager $m$ are eventually purged from the registered services of Registry, c. If an entity is both a Registry and a User then both properties must hold.

g) Uptodate $(\mathrm{u}, \mathrm{m}):=\operatorname{OfferedSD}(\mathrm{m}) \cap$

Requirement $(\mathrm{u}) \diamond \subseteq \operatorname{DiscoveredSD}(\mathrm{u}, \mathrm{m}) \wedge$

OfferedSD $(\mathrm{m}) \diamond \supseteq \operatorname{DiscoveredSD}(\mathrm{u}, \mathrm{m})$

states that User $u$ will find new services at Manager $\mathrm{m}$ and remove services that are no longer available at $\mathrm{m}$.

Figure 1 and 2 provides scenarios of a registry-based system, where the relationship between entities, and the sets are explained, as time progresses. In Figure 1, ServiceSearch is the message providing the requirements of the User to the Registry, while in Figure 2, PurgeService and PurgeService' are the messages that notify the Registry and User respectively of a defunct service.

With the system and basic properties in place we are ready to give the 7 Service Discovery Principles.

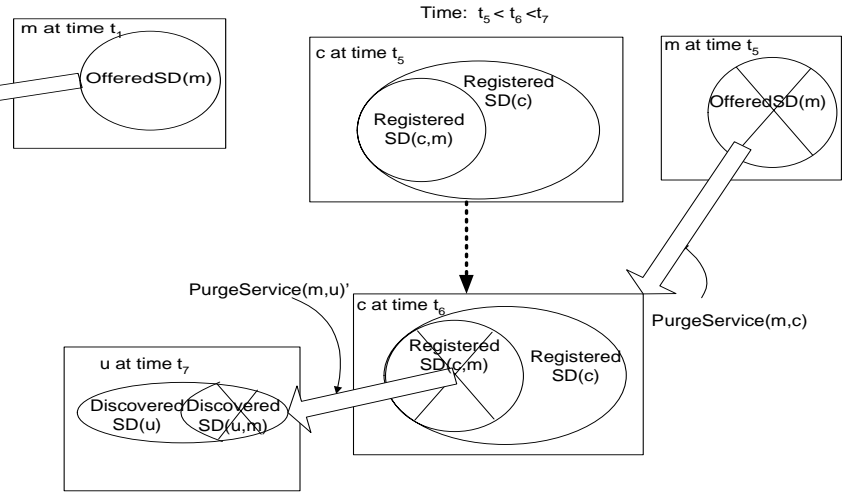

Fig. 2. System flow and relations between sets during Configuration Purge. A service is purged by the Manager at $t_{5}$, then the Registry is notified at $t_{6}$, which then purges the registration. The Registry notifies the User at $\mathrm{t}_{7}$, and the User purges the service description from its DiscoveredSD cache.

\section{(P1) Registry Setup Principle}

When there is global connectivity, N Registries of the highest rank are selected in the system.

$\square(\mathrm{GC} \rightarrow \diamond((|\mathrm{C}|=\mathrm{N}) \wedge$ highestRank $))$

Assume all entities have the same rank if no ranking function is used.

(P2) Registry Discovery Principle

An entity discovers all available Registries in the system that it is interested in.

$\forall \mathrm{e}: \square(\mathrm{C}(\mathrm{e}) \subseteq \mathrm{G}(\mathrm{e}) \wedge$

$$
\forall c \in C \cap \mathrm{G}(\mathrm{e}): \text { Conn }(\mathrm{c}, \mathrm{e}) \rightarrow \diamond \mathrm{c} \in \mathrm{C}(\mathrm{e}))
$$

(P3) Registration Principle

A Manager registers its service description at each Registry it discovers.

$\forall \mathrm{m}, \forall \mathrm{c} \in \mathrm{C}(\mathrm{m})$ :

$\square($ Conn $(\mathrm{m}, \mathrm{c}) \rightarrow$ Registration $(\mathrm{m}, \mathrm{c}))$

(P4) Registry-based Service Discovery Principle A User discovers the services that match the User's requirements and which are registered at a Registry. $\forall \mathrm{u}, \forall \mathrm{c} \in \mathrm{C}(\mathrm{u}):$

$$
\square(\text { Conn }(\mathrm{u}, \mathrm{c}) \rightarrow \text { ServiceFound }(\mathrm{c}, \mathrm{u}))
$$

(P5) Configuration Purge Principle

A User or Registry purges the services of a Manager that has become disconnected.

$\forall \mathrm{m}, \forall \mathrm{uc} \in \mathrm{U} \cup \mathrm{C}$ :

$\square(\operatorname{Dis}$ Conn $(\mathrm{m}, \mathrm{uc}) \rightarrow$ PurgeService $(\mathrm{m}, \mathrm{uc}))$

(P6) 2-Party Configuration Update Principle: A User remains consistent with a Manager when its services change.

$\forall \mathrm{m}, \mathrm{u}: \square(\operatorname{Conn}(\mathrm{u}, \mathrm{m}) \rightarrow \operatorname{Uptodate}(\mathrm{u}, \mathrm{m}))$

(P7) 3-Party Configuration Update Principle

A User remains consistent with a Manager when its services change, through the Registry.

$\forall \mathrm{c} \in \mathrm{C}(\mathrm{m}): \square((\operatorname{Conn}(\mathrm{c}, \mathrm{m}) \wedge \diamond \operatorname{Conn}(\mathrm{u}, \mathrm{c})) \rightarrow$ Uptodate $(\mathrm{u}, \mathrm{m}))$

The 7 Service Discovery Principles above state the precise behavior of all the functions in service 
discovery. The Service Discovery Principles state the fundamental objectives of service discovery; the User discovers an available Manager, which matches its service requirement, and achieve consistency with the Manager when the service changes.

\section{FRODO}

FRODO implements resource-awareness and robustness. For resource-awareness, FRODO classifies nodes as (1) 3C device class - simple devices with restricted resources (e.g. smart dust), (2) 3D device classmedium complex devices (e.g. temperature controller) and (3) 300D device class - powerful devices, controlled by a complex embedded computer, with more than 1MB memory requirement (e.g. set-top boxes). Only a 300D node can become a Registry, through a leader election process. The Registry is called the Central. The functions of service discovery explained in Section II-D are implemented according to the device classes. The names of the functions remain the same for FRODO, except we classify Configuration Update as part of the wider Configuration Management, to accommodate its robust features. Table I provides a summary of the mechanisms that each function implements. The protocol is less dependent than the state-of-the-art on the recovery ability of the lower layers. This allows the protocol to be deployed together with leaner lower layer protocol stacks, with restricted error recovery mechanisms. More details on FRODO can be found in [6].

\section{Modeling AND VERIFICATION}

We use model checking [9] to verify that FRODO adheres to the Service Discovery Principles. If there are cases where the protocol fails the verification, we identify whether the error lies in the modeling process, or in the design phase. The former requires the model to be corrected (and often our understanding!). However, it is the latter which is most beneficial, since detecting a design flaw leads towards discovering mechanisms that improve and strengthen the protocol, until the Service Discovery Principles are satisfied.

We verify the desired behavioral properties of the protocol through exhaustive enumeration (explicit or implicit) of all the states reachable by the system and the behaviors that navigate through them. We use the model-checker DT-Spin [10], for this purpose. DTSpin is an extension of the well-known SPIN tool [9]. DT-Spin is used instead of the original SPIN model checker because we need multiple timeouts that occur at any time, even if other processes are being executed in the model. Timeouts are essential requirements for the recovery processes of the protocol against failures. The timeout variable in standard SPIN cannot be used as it is only true when the system is idle. However, DT-Spin also increases state space because time is

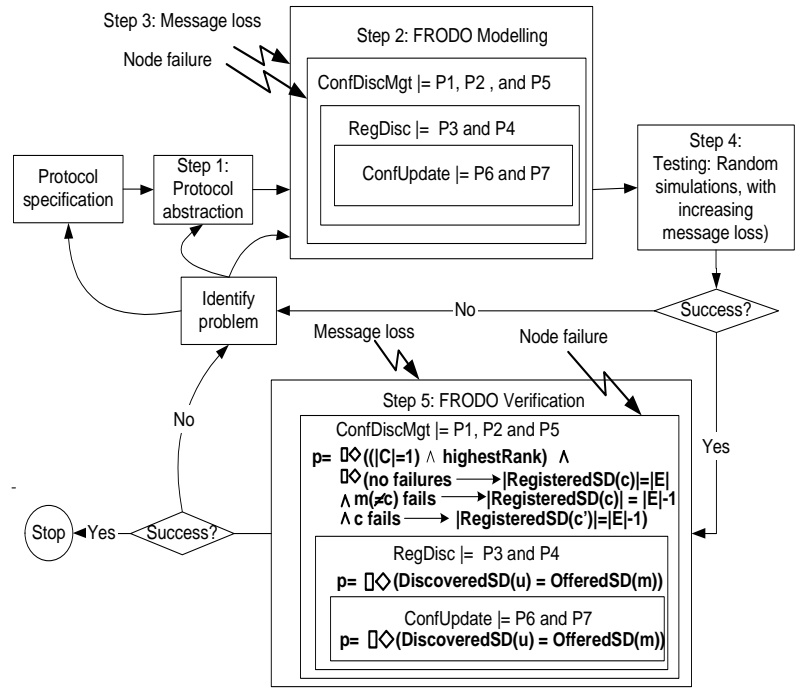

Fig. 3. Methodology steps. The FRODO Modeling box shows the abstraction link between the 3 modules. The SrvRegDisc module abstracts some functions in the ConfDiscMgt module, while the ConfUpdate module abstracts some functions in the ConfDiscMgt and SrvRegDisc modules. Connectivity and Global Connectivity are modeled with/without message loss respectively, and Disconnect is modeled as node failure.

modeled as ticks, and each tick occupies a state. A reasonable number of ticks is set for each timer, to make verification feasible.

\section{A. Methodology}

It is impractical to work with monolithic models of complex protocols such as FRODO. In the first place error traces would be too cluttered with irrelevant detail to be able to spot the real problems, and secondly the state space would grow beyond the bounds of what the current tools can cope with. Therefore we split the FRODO protocol in a number of modules, each of which corresponds roughly to one of the four functional areas or sub-functions thereof. Each model is then provided in several versions, including a concrete model with the most detail, some versions that represent worst case behavior, and a number of abstract versions with as little detail as possible. By combining a concrete version of one module with appropriate abstract versions of all others, the system as a whole can be verified, focusing on the behavior of the concrete module. The main disadvantage of this method is to keep the different versions of each module consistent. Figure 3 shows the methodology that we use to model, simulate, and verify FRODO against the Service Discovery Principles.

a) Step 1: Protocol abstraction: . Each assembly of modules builds on a common layer of protocol abstraction. We deliberately abstract irrelevant detail such as message format.

b) Step 2: Modular decomposition.: a) ConfDiscMgt models Configuration Discovery and Configuration Management. This part is actually broken into 
four sub-functions as follows: ConfDiscMgt-1 models the leader election protocol which elects one Central. ConfDiscMgt-2 models two worst-case scenarios of the protocol with (a) all nodes claiming to be Central after a network partitioning, and (b) all registration information in the Central being lost because of prolonged communication failure.

ConfDiscMgt-3 models a Backup taking over as the Central when the existing Central leaves the system. This model uses node failure, instead of message loss to model network disturbance. ConfDiscMgt-4 models the Central handing over to a superior node that enters late into the system. This model is an abstraction of ConfDiscMgt-1, and built without message loss, to ensure that the handover protocol is correct.

b) SrvRegDisc models both Registration and Service Discovery functions. Configuration Discovery is abstracted away in this model. The model consists of one 300D node which is the Central. Service discovery is done through the Central using the directed search mechanism, thus the service cannot be discovered unless registration occurs. The discovery of each type of Manager (3C, 3D and 300D) is modelled separately. c) ConfUpdate models Configuration Update propagating the updates to the Users through the 2-party and 3-party subscription mechanisms.

c) Step 3: Message loss.: Network disturbance is modeled as message loss. All models except ConfDiscMgt-3 and ConfDiscMgt-4 are simulated and verified with and without message loss. We allow the sender to lose or send the message instead of modeling message loss as premature timeouts [11], because premature timeouts focuses on dropping acknowledgement messages, which leads to the timeout of the waiting entity. All messages, not only acknowledgement messages can be lost in our models. The targeted receiver will continue executing its tasks, unaware of any message loss, which may lead towards a violation of the Service Discovery Principles. We use a counter, lossCounter for every message type, which increments whenever a particular type of message is lost. The message may be lost, until a constant MAX_LOSS for its type is reached. The following is an example of how a SrvRegReq message is sent or lost. The variables rcvrID, OfferedSD, and srcID are the receiver node's identifier, the service identifier that represents a service and the sender node's identifier respectively.

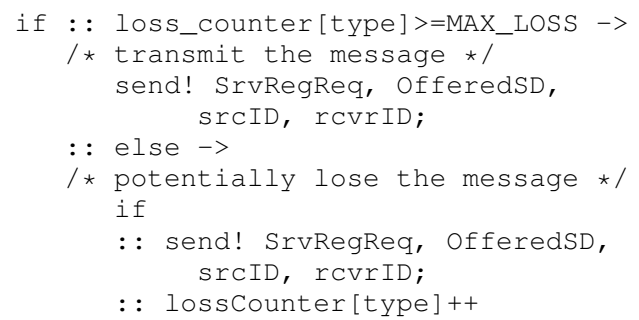

\begin{tabular}{|l|l|l|l|}
\hline Model & $\begin{array}{l}\text { State } \\
\text { vector } \\
\text { (bytes) }\end{array}$ & Depth & States stored \\
\hline $\begin{array}{l}\text { ConfDiscMgt-1, ex- } \\
\text { haustive mode with- } \\
\text { out message loss }\end{array}$ & 304 & 37328 & 38345 \\
\hline $\begin{array}{l}\text { ConfDiscMgt-1, } \\
\text { supertrace mode, } \\
\text { MAX_LOSS=1 }\end{array}$ & 308 & 61993 & $4,976,620$ e+07 \\
\hline
\end{tabular}

TABLE II

An example of the impact of message loss on state space. The rest of the models reflect similar increase in state space.

Message loss increases the number of states because of the additional counter and timing variables and nondeterministic choice. The impact of message loss on the verification is shown in Table II.

d) Step 4: Testing.: We use the simulator tool in DT-Spin to test and debug the models. We test different, random simulation scenarios and increase the MAX_LOSS for every simulation (up to 10 message losses to capture extreme scenarios)

e) Step 5: Verification.: DT-Spin checks correctness claims that are generated from logic formulas expressed in LTL. When a claim is invalid for a model, the tool produces a counter example that explicitly shows how the property was violated. The counter example is a feedback for the simulator tool of DT-Spin to trace the execution trail that causes the violation. The next section describes the properties.

\section{B. Property Modeling}

The following are the interpretation of the protocol as described in Section II-C (protocol-dependent parameters):

1) Connectivity condition: Conn $\left(\mathrm{e}, \mathrm{e}^{\prime}\right)$ : if a message is transmitted from either e to $\mathrm{e}^{\prime}$, or vice versa, and the expected acknowledgement arrives, the entities are reachable.

2) Disconnect condition: $\neg$ Conn $\left(e, e^{\prime}\right)$ : for twice the timeout period.

3) Rank, a leader election function that returns the highest ranked 300D node as the Central.

4) $\mathrm{G}(\mathrm{e})=\mathrm{E}$ the nodes are interested in any Central as there is only one type of Central in Frodo.

5) $\mathrm{N}=1$, a single Central is elected.

We model the response pattern $\square\left(p^{\prime} \rightarrow \diamond p\right)$ defined in Section II-D by building the property $p^{\prime}$ directly into the models, so that $\mathrm{p}$ is verified as a recurrence property [9]. The recurrence property $\square \diamond \mathrm{p}$ states that if the state formula, $\mathrm{p}$ happens to be false at any given point in a run, it is always guaranteed to become true again if the run is continued. For $\mathrm{p}^{\prime}=$ Global Connectivity, we verify $\mathrm{p}$ in a model without any message loss. For $\mathrm{p}^{\prime}=$ Connectivity, we verify $\mathrm{p}$ in a model with a limit on message loss. For $p^{\prime}=$ Disconnect, we verify $p$ in a model with node failure. 
The descriptions of each property, $\mathrm{p}$ are given below. For the purpose of simplification and readability, we left out some technical details. The properties are summarized in Figure 3.

\section{ConfDiscMgt $\models \mathrm{P} 1 \wedge \mathrm{P} 2 \wedge \mathrm{P} 5$}

For $\mathrm{P} 1$, all entities have to agree on a the highest ranking node, say c, becoming the single Central. For P2, all nodes must discover this Central (and no others). In the verification we check $|\operatorname{RegisteredSD}(\mathrm{c})|=|\mathrm{E}|$ which implies that each node $\mathrm{m}$ has registered at $\mathrm{c}$ As each node will offer exactly one service, it will only discover one Central and will only register at this Central. For P5, the Central purges service registration of disconnected nodes. Thus, $\mid$ RegisteredSD $(\mathrm{c}) \mid=$ $|\mathrm{E}|-1$. If the failing node is the Central itself then the Backup $c^{\prime}$, which is the second highest ranking node, must detect this and take over as the new Central, resulting in $\mid$ RegisteredSD $\left(c^{\prime}\right)|=| E \mid-1$. Thus we check property

$\mathrm{p}:=\square \diamond($ no failures $\rightarrow \mid$ RegisteredSD $(\mathrm{c})|=| \mathrm{E} \mid \wedge$

$\mathrm{m}(\neq \mathrm{c})$ fails $\rightarrow \mid$ RegisteredSD $(\mathrm{c})|=| \mathrm{E} \mid-1 \wedge$

$\mathrm{c}$ fails $\rightarrow \mid$ RegisteredSD $\left.\left(\mathrm{c}^{\prime}\right)|=| \mathrm{E} \mid-1\right)$

Together with some basic properties of the behavior of each of the nodes (e.g. discovering only one Central) only registering at a node it believes to be the Central, ...) this is sufficient to obtain that P1, P2 and P5 all hold.

\section{SrvRegDisc $=\mathrm{P} 3 \wedge \mathrm{P} 4$}

We consider the situation where a User $u$ is interested in the service that is offered by some Manager $m$ in the system, Requirement $(\mathrm{u})=\operatorname{OfferedSD}(\mathrm{m})$. We verify the property

$\mathrm{p}:=\square \diamond(\operatorname{DiscoveredSD}(\mathrm{u})=\operatorname{OfferedSD}(\mathrm{m}))$

Which gives that services can be found $(\mathrm{P} 4)$ and also that services are registered (P3) as the User can only discover registered services.

\section{ConfUpdate $=\mathrm{P} 6 \wedge \mathrm{P} 7$}

We consider the situation where the service at $\mathrm{m}$ discovered by a User $u$ changes but still satisfies the requirements of the User. To satisfy $\mathrm{P} 6$ and $\mathrm{P} 7$, the User has to update its discovered services to remain consistent with the Manager, Uptodate $(u, m)$. This is implied by DiscoveredSD $(\mathrm{u})=\operatorname{OfferedSD}(\mathrm{m})$. We again check the property

$\mathrm{p}:=\square \diamond(\operatorname{DiscoveredSD}(\mathrm{u})=\operatorname{OfferedSD}(\mathrm{m}))$

now, however, OfferedSD $(\mathrm{m})$ changes during the verification run.

\section{RESUlTS \& Discussion}

\section{A. Verification results}

The results that we obtained are summarized in Table III. For every module, we provide the setting for the verification. Note that there are multiple MAX_LOSS values. This is because for every module, there are several models, which we verified for the same properties. In total, we developed and verified 21 models, out of which, 17 models achieved Exhaustive coverage (100\% coverage of all reachable states for a model), while 4 models had to be run under the Supertrace mode (uses bit state hashing [12], with coverage around $98 \%$ ) because of state space explosion.

The successful results shown in Table III are under the assumptions that (1) the nodes provide correct information, (2) message losses are low, and (3) the system does not require a fixed time constraint on satisfying the Service Discovery Principles. Based on these assumptions, the results show that FRODO guarantees that the functions of service discovery meet their objectives under the condition that nodes are able to communicate eventually. The results of the verification increases confidence in FRODO's capabilities.

\section{B. Analyzing Service Discovery Behavior}

In service discovery, the system can oscillate from an ideal state, to a non-ideal state, for every service discovery function, where a network disturbance is the trigger for the transition. For example, a node successfully discovers the Registry, but the registration is unsuccessful because the registration message is lost. Thus the Registry Setup and Registry Discovery Principles are satisfied, but the Registration Principle is violated. The service discovery protocol must ensure that the Registration function can return to the ideal state, and satisfy the principle.

Several mechanisms are vital to satisfy the Service Discovery Principles. These are: (1) periodic lazy discovery - periodic announcements from the Registries ensure detection of other Registries, to recover from network partitioning. A service discovery system can use this scheme to either converge into N Registries, or Registries can synchronize their configuration information. (2) Re-registration - a previous successful registration does not necessarily mean it remains valid. The Registry can face node failure, or can purge the knowledge of the Manager because of failures on the Manager, Registry or the communication paths. There are several mechanisms that can resolve this issue: (a) periodic leasing with the Registry, a popular concept for most service discovery systems. The use of this method alone violates the Registration and Registrybased Service Discovery Principles if the Registry has node failure, and the Manager does not attempt to register with any other available Registries, (b) Registry piggybacks request for re-registration, in its periodic lazy announcement. Nodes monitor Registry announcements to detect an unknown Registry, and initiate unsolicited registration, and (c) the Registry monitors periodic active announcements of the nodes to detect unknown nodes. Unknown nodes are requested to register through solicited registration. (3) 


\begin{tabular}{|c|c|c|c|}
\hline Module & Settings & Principle & Results \\
\hline $\begin{array}{l}\text { ConfDiscMgt, without solicited } \\
\text { registration and lazy discovery }\end{array}$ & $\begin{array}{l}\text { MAX_LOSS }=1 \text { to } 4, \mathrm{~N}=1, \mathrm{E}=4 \\
\text { for ConfDiscMgt- } 1 \text { and ConfDiscMgt- } \\
2 \mathrm{E}=3 \text { for ConfDiscMgt- } 3 \text { and } \mathrm{E}=5 \\
\text { for ConfDiscMgt- } 4\end{array}$ & $\begin{array}{l}\text { Registry Setup, Registry } \\
\text { Discovery, Configuration } \\
\text { Purge }\end{array}$ & Fail \\
\hline $\begin{array}{l}\text { ConfDiscMgt, with solicited reg- } \\
\text { istration and lazy discovery }\end{array}$ & $\begin{array}{l}\text { MAX_LOSS }=1 \text { to } 4, \mathrm{~N}=1, \mathrm{E}=4 \\
\text { for ConfDiscMgt- } 1 \text { and ConfDiscMgt- } \\
2, \mathrm{E}=3 \text { for ConfDiscMgt- } 3 \text { and } \mathrm{E}= \\
5 \text { for ConfDiscMgt- } 4 \text {, }\end{array}$ & $\begin{array}{l}\text { Registry Setup, Registry } \\
\text { Discovery, Configuration } \\
\text { Purge }\end{array}$ & Success \\
\hline SrvRegDisc & $\begin{array}{l}\text { Nodes }=3 \text { (Central, Manager, User), } \\
\text { MAX_LOSS }=1, \mid \text { OfferedSD }(\mathrm{m}) \mid=1\end{array}$ & $\begin{array}{l}\text { Registration, Service Dis- } \\
\text { covery }\end{array}$ & Success \\
\hline ConfUpdate & $\begin{array}{l}\text { Nodes=4 (Central, Manager, User), } \\
\text { MAX_LOSS }=1, \mid \text { OfferedSD }(\mathrm{m})=1 \mid \text {, } \\
\text { then } 2,\end{array}$ & Configuration Update & Success \\
\hline $\begin{array}{l}\text { Verify models during uncon- } \\
\text { nected state }\end{array}$ & $\begin{array}{l}\text { Nodes=4 300Ds, message loss not lim- } \\
\text { ited }\end{array}$ & $\begin{array}{l}\text { Disconnect check on all } \\
\text { principles. }\end{array}$ & $\begin{array}{l}\text { Success. Principles } \\
\text { still hold }\end{array}$ \\
\hline
\end{tabular}

TABLE III

Verification results. Failures are corrected by implementing additional mechanisms

Ensuring update propagation - acknowledgements can help in ensuring an update is propagated successfully. However, if the Manager undergoes another change before the first update is propagated, it is likely that the Registry and the User will never be aware of the first change. Polling the Manager when an expected update is missed (e.g. through message sequence) ensures that the updates are always propagated successfully.

\section{COnClusion And Future Work}

We classify the behavior of registry-based service discovery into 4 functional areas, and analyze the mechanisms used in each of these areas. We show that existing registry based service discovery protocols fit into this classification.

The functional requirements of service discovery, needed for correct behaviour of applications in the ubiquitous environment, are expressed in 7 Service Discovery Principles. Existing protocols do not satisfy these principles because they rely on the underlying network to provide robustness. In contrast, our own service discovery protocol FRODO does satisfy the principles. We show this by formal modeling and verification.

FRODO is the first service discovery protocol that provides formally verified functional guarantees. The simulation and verification process is beneficial in discovering flaws in the design and identifying essential mechanisms needed to satisfy the Service Discovery Principles.

Future work includes investigating the nonfunctional aspects of service discovery, such as time constraints.

\section{ACKNOWLEDGEMENT}

This research is sponsored by the Netherlands Organization for Scientific Research (NWO) under grant number 612.060.111, and by the IBM Equinox program. We thank K.Mills and C.Dabrowski from the National Institute of Standards and Technology
(NIST), Gaithersburg, MD for their contributions to this work.

\section{REFERENCES}

[1] K. Arnold, R. Scheifler, J. Waldo, B. O'Sullivan, and A. Wollrath, The Jini Specification, V1.1, 1999.

[2] Microsoft, Universal Plug and Play Architecture, V1.0, Jun 2000.

[3] C. Bettstetter and C. Renner, "A comparison of service discovery protocols and implementation of the service location protocol," in Proceedings of 6th EUNICE Open European Summer School: Innovative Internet Applications, September 2000.

[4] E. Guttman, C. Perkins, J. C. Veizades, and M. Day, Service Location Protocol, V.2, December 2003.

[5] J. Bray, C. F. Sturman, and J. Mandolia, Bluetooth 1.1 Connect Without Cables, 2nd Edition. Prentice Hall, December 2001.

[6] V. Sundramoorthy, J. Scholten, P. G. Jansen, and P. H. Hartel, "Service discovery at home," in 4th Int. Conf. on Information, Communications \& Signal Processing and 4th IEEE PacificRim Conf. On Multimedia (ICICS/PCM). IEEE Computer Society Press, December 2003.

[7] C. Dabrowski, K. Mills, and S. Quirolgico, A Model-based Analysis of First-Generation Service Discovery Systems. Special Publication 500-260, National Institute of Standards and Technology, 2005.

[8] M. Huth and M. Ryan, "Logic in computer science: Modelling and reasoning about systems." Cambridge University Press, First Edition, January 2000.

[9] G.J.Holzmann, "The model checker spin, primer and reference manual." Addison-Wesley, September 2003.

[10] D. Bosnacki, "Implementing discrete time in promela and spin," in Proceedings of the VIII Conference on Logic and Computer Science, LIRA '97,, 1997, pp. 25-32.

[11] T. Ruys and R.Langerak, "Validation of bosch' mobile communication network architecture with spin," in Proc. SPIN97, the Third International Workshop on SPIN, April 1997.

[12] G.J.Holzmann, "An analysis of bitstate hashing," in Protocol Specification, Testing and Verification. 15th International Conference. Kluwer Academic Publishers, November 1998. 